PROCEEDINGS OF THE

AMERICAN MATHEMATICAL SOCIETY

Volume 126, Number 5, May 1998, Pages 1397-1407

S 0002-9939(98)04237-3

\title{
DITKIN'S CONDITION FOR CERTAIN BEURLING ALGEBRAS
}

\author{
SEN-ZHONG HUANG, JAN VAN NEERVEN, AND FRANK RÄBIGER \\ (Communicated by Palle E. T. Jorgensen)
}

\begin{abstract}
Let $G$ be a locally compact abelian group. A function $\omega: G \rightarrow$ $[1, \infty)$ is said to be a weight if it is locally bounded, Borel measurable and submultiplicative. We call a weight $\omega$ on $G$ semi-bounded if there exist a constant $K$ and a subsemigroup $S$ with $S-S=G$, such that

$$
\omega(s) \leq K \quad \text { and } \quad \lim _{n \rightarrow \infty} \frac{\log \omega(-n s)}{\sqrt{n}}=0
$$

for all $s \in S$. Using functional analytic methods, we show that all Beurling algebras $L_{\omega}^{1}(G)$ whose defining weight $\omega$ is semi-bounded satisfy Ditkin's condition.
\end{abstract}

\section{INTRODUCTION}

Let $\mathcal{A}$ be a commutative Banach algebra and denote by $\Delta(\mathcal{A})$ its Gelfand space. $\mathcal{A}$ is called regular if for every closed subset $E \subset \Delta(\mathcal{A})$ and every point $M \notin E$ there exists an element $f \in \mathcal{A}$ such that $\breve{f} \equiv 0$ on $E$ and $\breve{f}(M) \neq 0$, where $\breve{f}$ is the Gelfand transform of $f$. We say that $\mathcal{A}$ satisfies Ditkin's condition at $M \in \Delta(\mathcal{A})$ if for each $f \in \mathcal{A}$ with $\breve{f}(M)=0$ there exists a sequence $\left(f_{n}\right) \subset \mathcal{A}$ such that $\breve{f}_{n} \equiv 0$ in a neighbourhood $V_{n}$ of $M$ and $f f_{n} \rightarrow f$ in the norm of $\mathcal{A}$. Further, $\mathcal{A}$ satisfies Ditkin's condition at infinity if the subalgebra of all $f \in \mathcal{A}$ whose Gelfand transforms $\breve{f}$ have compact support is norm dense in $\mathcal{A}$. The algebra $\mathcal{A}$ is called a Ditkin algebra if it satisfies Ditkin's condition at every point of $\Delta(\mathcal{A})$ and at infinity.

The importance of the Ditkin condition resides in the well-known fact that closed subsets with scattered boundary of the maximal regular ideal space of a regular semisimple Banach algebra satisfying the Ditkin condition are sets of spectral synthesis (see [12, p.225], [14, p.86] and [8]). Recently, Bade and Dales have found its applications to the automatic continuity of epimorphisms (see [5, Theorem 2.3]).

The group algebra $L^{1}(G)$ of a locally compact abelian (LCA) group $G$ is a Ditkin algebra. This is a standard result in harmonic analysis; apart from this example however, only relatively few subalgebras of $L^{1}(G)$ are known to satisfy Ditkin's condition, e.g.

- the Segal algebras [17, pp. 92-93];

Received by the editors October 14, 1996.

1991 Mathematics Subject Classification. Primary 43A45, 43A20, 47D03.

Key words and phrases. Ditkin's condition, group representation, spectrum.

This research is supported by Deutscher Akademischer Austauschdienst DAAD (first author) and by the Human Capital Mobility Programme of the European Community (second author). It is part of a research project supported by Deutsche Forschungsgemeinschaft DFG (third author). 
- the Beurling algebras $L^{1}\left(\mathbb{R}^{n} ; \omega_{\alpha}\right)$ and $L^{1}\left(\mathbb{Z}^{n} ; \omega_{\alpha}\right)$, where $0 \leq \alpha<1$ and $\omega_{\alpha}\left(t_{1}, \ldots, t_{n}\right):=\left(\sum_{j=1}^{n} t_{j}^{2}\right)^{\alpha / 2}$ (see [16, p. 132 and p.137]);

- the Beurling algebras $L_{\alpha}^{1}\left(\mathbf{Q}_{l}^{v}\right)$, where $\mathbf{Q}_{l}^{v}$ is the $v$-times product of the group $\mathbf{Q}_{l}$ of $l$-adic numbers and $\alpha \geq 0$ (see [16, pp. 134-136]);

- the Beurling algebras $L^{1}(\mathbb{R} ; \omega)$ and $L^{1}(\mathbb{Z} ; \omega)$, where the weight $\omega$ is uniformly bounded on $[0,+\infty)$ and $\lim _{n \rightarrow+\infty} n^{-\frac{1}{2}} \log \omega(-n)=0$ (see [20, Cor. 3.5]).

For all these algebras the Ditkin condition was checked by classical methods involving the construction of functions with certain special properties. In this paper, we will extend this list by introducing axiomatically a class of subalgebras of $L^{1}(G)$ whose members turn out to be Ditkin algebras. This class contains the Segal algebras and many Beurling algebras that were not previously known to satisfy Ditkin's condition. Our approach is novel, and is based on functional analytic methods.

The paper is organized as follows. In Section 1 we recall some facts from the spectral theory of non-quasianalytic group representations. In Section 2 we prove our main results.

\section{Spectral theory of NON-Quasianalytic Representations OF LCA GROUPS}

In this section we briefly recall some well-known facts concerning measure algebras, Beurling algebras, and spectral theory of representations of LCA groups on a Banach space. We refer to [10], [14], [12] or [3] for the basic theory of LCA groups and Banach algebras.

Let $G$ be an LCA group with unit element $e$. A weight $\omega$ on $G$ is a function $\omega: G \rightarrow[1, \infty)$ which is locally bounded, Borel measurable and submultiplicative in the sense that

$$
\omega(s+t) \leq \omega(s) \omega(t) \quad \text { for all } \quad s, t \in G .
$$

Let $M(G)$ be the measure algebra of all bounded, regular Borel measures on $G$, with convolution as multiplication. For a weight $\omega$ on $G$, the subalgebra $M_{\omega}(G)$ is defined as

$$
M_{\omega}(G):=\left\{\mu \in M(G):\|\mu\|_{\omega}:=\int_{G} \omega(t) d|\mu|(t)<\infty\right\}
$$

and the Beurling algebra $L_{\omega}^{1}(G)$ is defined as

$$
L_{\omega}^{1}(G):=\left\{f \in L^{1}(G):\|f\|_{\omega}:=\int_{G}|f(t)| \omega(t) d t<\infty\right\} .
$$

It follows from Lebesgue's decomposition theorem that $L_{\omega}^{1}(G)$ is identical to the absolutely continuous part of $M_{\omega}(G)$, and thus $L_{\omega}^{1}(G)$ is an ideal of $M_{\omega}(G)$. It is shown by Domar [9, Theorem 2.11] that $L_{\omega}^{1}(G)$ is a regular Banach algebra if and only if the weight $\omega$ is non-quasianalytic (n.q.a.), i.e.,

$$
\sum_{n=1}^{\infty} \frac{\log \omega(n t)}{n^{2}}<\infty \text { for all } t \in G .
$$

This generalizes a theorem of Beurling $[4$, Theorem V B] for $G=\mathbb{R}$. It follows that if $\omega$ is an n.q.a. weight on $G$, then the ideal

$$
K_{\omega}(G):=\left\{f \in L_{\omega}^{1}(G): \hat{f} \text { has compact support }\right\}
$$


is norm dense in $L_{\omega}^{1}(G)$. Here, as usual, $\hat{f}$ is the Fourier transform of $f \in L_{\omega}^{1}(G)$,

$$
\hat{f}(\gamma):=\int_{G} \gamma(t) f(t) d t \quad \text { for all } \quad \gamma \in \hat{G},
$$

where $\hat{G}$ is the dual group of $G$.

Let $X$ be a (complex) Banach space. We denote by $\mathcal{L}(X)$ the Banach space of all bounded linear operators on $X$. A continuous algebra homomorphism $\Phi$ : $M_{\omega}(G) \rightarrow \mathcal{L}(X)$ is called a non-quasianalytic representation if the defining weight $\omega$ is non-quasianalytic and $\Phi$ maps the unit of $M_{\omega}(G)$ to the identity operator $I_{X}$ on $X$. A systematic theory of n.q.a. representations has been developed recently by one of the authors [11]. Here, we collect some results from this theory needed for our present purposes.

Let $\Phi: M_{\omega}(G) \rightarrow \mathcal{L}(X)$ be an n.q.a. representation. The set

$$
I_{\Phi}:=\left\{f \in L_{\omega}^{1}(G): \Phi_{f}=0\right\}
$$

is a closed ideal in $L_{\omega}^{1}(G)$. The spectrum $\operatorname{Sp}(\Phi)$ of $\Phi$ is defined as the hull $\mathrm{h}\left(I_{\Phi}\right)$ of $I_{\Phi}$, i.e.

$$
\operatorname{Sp}(\Phi):=\mathrm{h}\left(I_{\Phi}\right)=\left\{\gamma \in \hat{G}: \hat{f}(\gamma)=0 \quad \text { for all } \quad f \in I_{\Phi}\right\} .
$$

For a closed subset $\Lambda \subset \hat{G}$ we denote by $j_{\omega}(\Lambda)$ and $k_{\omega}(\Lambda)$ the following ideals in $L_{\omega}^{1}(G)$ :

$$
j_{\omega}(\Lambda):=\left\{f \in L_{\omega}^{1}(G): \hat{f} \text { vanishes on a neighbourhood of } \Lambda\right\}
$$

and

$$
k_{\omega}(\Lambda):=\left\{f \in L_{\omega}^{1}(G): \hat{f} \text { vanishes on } \Lambda\right\} .
$$

If $\omega$ is bounded, then the subscript $\omega$ will be omitted and the ideals are taken in $L^{1}(G)$.

An n.q.a. representation $\Phi: M_{\omega}(G) \rightarrow \mathcal{L}(X)$ is called non-degenerate if the kernel

$$
X_{0}:=\left\{x \in X: \Phi_{f}(x)=0 \text { for all } f \in L_{\omega}^{1}(G)\right\}
$$

is trivial, i.e., $X_{0}=\{0\}$.

Theorem 1.1 ([11, Cor.1.2.7 and Cor.1.3.17]). Let $\Phi: M_{\omega}(G) \rightarrow \mathcal{L}(X)$ be a nondegenerate n.q.a. representation. Then the following assertions hold:

(i) For all $f \in j_{\omega}(\mathrm{Sp}(\Phi))$ one has $\Phi_{f}=0$.

(ii) For all $t \in G$ one has

$$
\sigma\left(\Phi_{t}\right)=\overline{\{\gamma(t): \gamma \in \mathrm{Sp}(\Phi)\}}
$$

where $\Phi_{t}$ is the image of the Dirac measure $\delta_{t}$ under $\Phi$ and $\sigma\left(\Phi_{t}\right)$ is the spectrum of the operator $\Phi_{t}$.

The second assertion is referred to as "Spectral Mapping Theorem"; it extends a result of D'Antoni-Longo-Zsidó [6] for bounded representations.

We call a strongly continuous group representation $T: G \rightarrow \mathcal{L}(X)$ non-quasianalytic if there exists an n.q.a. weight $\omega$ on $G$ such that

$$
\left\|T_{t}\right\| \leq \omega(t) \text { for all } t \in G
$$


cf. [19]. For a strongly continuous n.q.a. group representation $T: G \rightarrow \mathcal{L}(X)$ we define the spectrum $\operatorname{Sp}(T)$ of $T$ as the set of all $\gamma \in \hat{G}$ for which there exists a net $\left\{x_{\alpha}\right\}$ of norm-one vectors in $X$ such that

$$
\left\|T_{t}\left(x_{\alpha}\right)-\gamma(t) x_{\alpha}\right\| \rightarrow 0
$$

uniformly for $t$ in compact subsets of $G$. The following functional calculus enables us to compute the spectrum of $T$ from an appropriate n.q.a. representation.

Theorem 1.2 ([11, Prop.1.3.3]). Let $T: G \rightarrow \mathcal{L}(X)$ be a strongly continuous n.q.a. group representation and let $\omega$ be an n.q.a. weight such that $\left\|T_{t}\right\| \leq \omega(t)$ for all $t \in G$. Then, for each $\mu \in M_{\omega}(G)$ there exists an operator $\Phi_{\mu} \in \mathcal{L}(X)$ such that

$$
\left\langle\rho, \Phi_{\mu}(x)\right\rangle=\int_{G}\left\langle\rho, T_{t}(x)\right\rangle d \mu(t) \quad \text { for all } \quad x \in X, \rho \in X^{*} .
$$

The map $\mu \mapsto \Phi_{\mu}$ defines a norm-decreasing algebra homomorphism $\Phi$ from $M_{\omega}(G)$ into $\mathcal{L}(X)$ which extends $T$ in the sense that $\Phi_{t}=T_{t}$ for all $t \in G$. Moreover, the spectrum $\operatorname{Sp}(\Phi)$ of $\Phi$ coincides with the spectrum $\operatorname{Sp}(T)$ of $T$.

Thus the spectral theory for n.q.a. group representations is an extension of Arveson's spectral theory for uniformly bounded group representations [1]. It easily follows from Theorem 1.2 that the functional calculus $\Phi$ of a strongly continuous n.q.a. group representation $T$ is non-degenerate. Thus by Theorem 1.1 (ii), the spectrum of $T$ is non-empty if the underlying Banach space $X$ is non-trivial. This fact is used in the proof of our main results below.

\section{The Ditkin condition for Certain subalgebras of $L^{1}(G)$}

Let $G$ be an LCA group. The right translation representation $\mathcal{R}$ of $G$ on $L^{1}(G)$ given by

$$
\mathcal{R}_{t} f(\cdot):=f(\cdot-t), \quad t \in G,
$$

is a strongly continuous representation and satisfies $\left\|\mathcal{R}_{t}\right\| \leq 1$ for all $t \in G$. Defining $\mathcal{R}_{\mu} f:=f * \mu, \mathcal{R}$ extends to a norm-decreasing algebra homomorphism from $M(G)$ to $\mathcal{L}(X)$, which will also be denoted by $\mathcal{R}$.

Definition 2.1. A weight $\omega$ on the LCA group $G$ is called semi-bounded if there exists a constant $K$ and a subsemigroup $S \subseteq G$ with $S-S=G$, such that

$$
\omega(s) \leq K \quad \text { and } \quad \lim _{n \rightarrow+\infty} \frac{\log \omega(-n s)}{\sqrt{n}}=0 \quad \text { for all } \quad s \in S .
$$

By definition, a semi-bounded weight is always non-quasianalytic.

Definition 2.2. Let $G$ be an LCA group and let $\mathcal{R}$ be the right translation representation of $G$ on $L^{1}(G)$. Let $\mathcal{S}(G)$ denote the class of all Banach subalgebras $\mathcal{A} \subset L^{1}(G)$ satisfying the following properties:

(i) $\mathcal{A}$ is norm dense in $L^{1}(G)$ and the injection $\mathcal{A} \hookrightarrow L^{1}(G)$ is continuous.

(ii) $\mathcal{A}$ is translation-invariant, i.e., $\mathcal{R}_{t} \mathcal{A} \subseteq \mathcal{A}$ for each $t \in G$.

(iii) For each $f \in \mathcal{A}$ the mapping $G \ni t \mapsto \mathcal{R}_{t} f \in \mathcal{A}$ is continuous.

(iv) There exists a semi-bounded weight $\omega$ on $G$ such that $\left\|\mathcal{R}_{t}\right\|_{\mathcal{A}} \leq \omega(t)$ for all $t \in G$.

(v) The intersection $\mathcal{A} \cap L_{\omega}^{1}(G)$ is norm dense in both $L_{\omega}^{1}(G)$ and $\mathcal{A}$. 
Example 2.3. (1) A subalgebra $\mathcal{A}$ of $L^{1}(G)$ is called a Segal algebra (see [17], [18]) if it satisfies the above properties (i)-(iii) and the following stronger version of (iv):

$$
\left\|\mathcal{R}_{t}\right\|_{\mathcal{A}}=1 \text { for all } t \in G \text {. }
$$

This trivially implies property (v). Consequently, all Segal algebras belong to the class $\mathcal{S}(G)$. The following are examples of Segal algebras (see [16], [17], [13]):

- The Wiener algebra

$$
W(\mathbf{R}):=\left\{f \in C_{b}(\mathbf{R}):\|f\|:=\sum_{n \in \mathbf{Z}} \sup _{x \in[n, n+1]}|f(x)|<\infty\right\} .
$$

- $L^{1}(G) \cap L^{p}(G)$ with the norm $\|f\|:=\|f\|_{1}+\|f\|_{p}, 1 \leq p<\infty$.

- $A_{p}(G):=\left\{f \in L^{1}(G): \hat{f} \in L^{p}(\hat{G})\right\}$ with the norm $\|f\|:=\|f\|_{1}+\|\hat{f}\|_{p}$, $1 \leq p<\infty$.

(2) A Beurling algebra $L_{\omega}^{1}(G)$ belongs to the class $\mathcal{S}(G)$ if and only if the defining weight $\omega$ is semi-bounded. To give a concrete example, let $\mathbf{d}:=\left(d_{1}, \ldots, d_{n}\right)$, where $0 \leq d_{j}<1 / 2$, and let $\omega_{\mathbf{d}}$ be the weight on $\mathbf{R}^{n}$ given by

$$
\omega_{\mathbf{d}}\left(t_{1}, \ldots, t_{n}\right):=\left\{\begin{array}{lc}
1, & t_{1}, \ldots, t_{n} \geq 0 \\
\exp \left(\sum_{j=1}^{n}\left|t_{j}\right|^{d_{j}}\right), & \text { otherwise }
\end{array}\right.
$$

The weight $\omega_{\mathbf{d}}$ is semi-bounded with constant $K:=1$ and generating subsemigroup

$$
S:=\left\{\left(t_{1}, \ldots, t_{n}\right) \in \mathbf{R}^{n}: t_{1}, \ldots, t_{n} \geq 0\right\} .
$$

Let $\mathcal{A}$ be in the class $\mathcal{S}(G)$, with corresponding semi-bounded weight $\omega$. The first three properties in Definition 2.2 imply that the right translation representation $\left\{\mathcal{R}_{t}: t \in G\right\}$ is a strongly continuous group representation of $G$ on $\mathcal{A}$ satisfying

$$
\left\|\mathcal{R}_{t}\right\|_{\mathcal{A}} \leq \omega(t) \text { for all } t \in G .
$$

It follows from Theorem 1.2 that $\mathcal{R}$ extends to an n.q.a. representation of $M_{\omega}(G)$ on $\mathcal{A}$, which will also be denoted by $\mathcal{R}$. For this representation we have

$$
\mathcal{R}_{\mu} f=f * \mu \quad \text { for all } \mu \in M_{\omega}(G), f \in \mathcal{A} .
$$

Therefore, for all $\mu \in M_{\omega}(G)$ and $f \in \mathcal{A}$,

$$
\begin{aligned}
\|f * \mu\|_{\mathcal{A}} & =\left\|\mathcal{R}_{\mu} f\right\|_{\mathcal{A}}=\left\|\int_{G} \mathcal{R}_{t} f d \mu(t)\right\|_{\mathcal{A}} \\
& \leq \int_{G}\|f\|_{\mathcal{A}} \cdot \omega(t) d|\mu|(t)=\|\mu\|_{\omega} \cdot\|f\|_{\mathcal{A}} .
\end{aligned}
$$

In particular, the extended algebra homomorphism $\mathcal{R}: M_{\omega}(G) \rightarrow \mathcal{L}(\mathcal{A})$ is contractive.

Proposition 2.4. Let $\mathcal{A}$ be a Banach algebra in the class $\mathcal{S}(G)$.

(i) For the Gelfand space of $\mathcal{A}$ we have $\Delta(\mathcal{A})=\hat{G}$.

(ii) $\mathcal{A}$ is regular and semisimple.

(iii) The set $\{f \in \mathcal{A}: \hat{f}$ has compact support $\}$ is dense in $\mathcal{A}$.

(iv) A closed subspace of $\mathcal{A}$ is an ideal if and only if it is translation-invariant.

(v) $f \in \overline{f * \mathcal{A}}$ for each $f \in \mathcal{A}$, i.e., $\mathcal{A}$ possesses approximate units.

Proof. Choose a semi-bounded weight $\omega$ corresponding to $\mathcal{A}$ as in Definition 2.2. Let $f \in L_{\omega}^{1}(G) \cap \mathcal{A}$ and denote by $f^{(n)}$ the $n$-fold convolution product of $f$. Then, $f^{(n)} \in L_{\omega}^{1}(G) \cap \mathcal{A}$ and $f^{(n+1)}=\mathcal{R}_{f}^{n} f$ for all $n$. Therefore, by $\left(^{*}\right)$

$$
\left\|f^{(n+1)}\right\|_{\mathcal{A}}=\left\|\mathcal{R}_{f^{(n)}} f\right\|_{\mathcal{A}} \leq\left\|f^{(n)}\right\|_{\omega} \cdot\|f\|_{\mathcal{A}} .
$$


Since

$$
\lim _{n \rightarrow \infty}\left\|f^{(n)}\right\|_{\omega}^{1 / n}=r(f):=\sup _{\gamma \in \hat{G}}|\hat{f}(\gamma)|
$$

we see that

$$
\lim _{n \rightarrow \infty}\left\|f^{(n)}\right\|_{\mathcal{A}}^{1 / n}=r(f) .
$$

Let $\psi \in \Delta(\mathcal{A})$. It follows that for all $f \in L_{\omega}^{1}(G) \cap \mathcal{A}$,

$$
|\psi(f)| \leq \lim _{n \rightarrow \infty}\left\|f^{(n)}\right\|_{\mathcal{A}}^{1 / n}=r(f) .
$$

By Definition $2.2(\mathrm{v}), L_{\omega}^{1}(G) \cap \mathcal{A}$ is norm dense in $L_{\omega}^{1}(G)$, and hence also in $L^{1}(G)$. Thus, the above inequality implies that $\psi$ extends to an element in $\Delta\left(L^{1}(G)\right)=\hat{G}$. This proves the inclusion $\Delta(\mathcal{A}) \subseteq \hat{G}$. The converse inclusion is obvious.

Recalling that the Gelfand tranform for $L^{1}(G)$ is just the Fourier transform, the semisimplicity in (ii) follows from the uniqueness theorem for the Fourier transform. To prove regularity, let $\gamma \in \hat{G}$ and $\Lambda \subset \hat{G}$ with $\gamma \notin \Lambda$. By the regularity of $L_{\omega}^{1}(G)$ we can find $f \in L_{\omega}^{1}(G)$ such that $\hat{f}(\gamma)=1$ and $\hat{f}_{\mid \Lambda} \equiv 0$. Since $\mathcal{A}$ is dense in $L^{1}(G)$ there exists $g \in \mathcal{A}$ such that $\hat{g}(\gamma)=1$. Consider $h:=f * g$. Then, $h=\mathcal{R}_{f} g \in \mathcal{A}$, $\hat{h}(\gamma)=1$ and $\hat{h}_{\mid \Lambda} \equiv 0$. This implies the regularity of $\mathcal{A}$.

For the proof of (iii) we note that the ideal $K_{\omega}(G)$ consisting of all $f \in L_{\omega}^{1}(G)$ whose Fourier transforms $\hat{f}$ have compact support is norm dense in $L_{\omega}^{1}(G)$ (see Section 1). We have $K_{\omega}(G) * \mathcal{A} \subseteq \mathcal{A}$, and the support of the Fourier transform of each function in $K_{\omega}(G) * \mathcal{A}$ is compact. Therefore, it suffices to show that $K_{\omega}(G) * \mathcal{A}$ is dense in $\mathcal{A}$. To this end, let $F \in\left(K_{\omega}(G) * \mathcal{A}\right)^{\perp}$. Then by the denseness of $K_{\omega}(G)$ in $L_{\omega}^{1}(G)$ we have $\langle F, f * g\rangle=0$ for all $f \in L_{\omega}^{1}(G)$ and $g \in \mathcal{A}$. Hence,

$$
0=\langle F, f * g\rangle=\left\langle F, \mathcal{R}_{f} g\right\rangle=\int_{G}\left\langle F, \mathcal{R}_{t} g\right\rangle f(t) d t \quad \text { for all } f \in L_{\omega}^{1}(G), g \in \mathcal{A} .
$$

It follows from the strong continuity of $\mathcal{R}$ that $\left\langle F, \mathcal{R}_{t} g\right\rangle=0$ for all $t \in G$ and $g \in \mathcal{A}$. This implies that $F=0$.

To prove (iv), let $I$ be a translation invariant closed subspace of $\mathcal{A}$. Let $f \in I$. Then $\mathcal{R}_{t}(f) \in I$ for all $t \in G$. This implies for all $g \in L_{\omega}^{1}(G) \cap \mathcal{A}$ that

$$
f * g=\mathcal{R}_{g} f=\int_{G} \mathcal{R}_{t}(f) g(t) d t \in I .
$$

Since $L_{\omega}^{1}(G) \cap \mathcal{A}$ is norm dense in $\mathcal{A}$ (Def. 2.2(v)), it follows that $f * g \in I$ for all $g \in \mathcal{A}$, and thus $I$ is a closed ideal of $\mathcal{A}$. For the converse assertion, let $I$ be a closed ideal of $\mathcal{A}$. To show the translation invariance of $I$ we first adopt the fact that $\mathcal{A}$ has approximate units, which will be immediately established in (v). Let $f \in I$. We choose $g_{n} \in \mathcal{A}$ such that $\left\|f-f * g_{n}\right\|_{\mathcal{A}} \rightarrow 0$ as $n \rightarrow \infty$. Let $t \in G$. Then

$$
\mathcal{R}_{t}(f)=\lim _{n \rightarrow \infty} \mathcal{R}_{t}\left(f * g_{n}\right)=\lim _{n \rightarrow \infty} f *\left(\mathcal{R}_{-t}\left(g_{n}\right)\right) .
$$

Since $\mathcal{R}_{-t}\left(g_{n}\right) \in \mathcal{A}$ and $I$ is an ideal, $f *\left(\mathcal{R}_{-t}\left(g_{n}\right)\right) \in I$ for all $n$. It follows from the closedness of $I$ that $\mathcal{R}_{t}(f) \in I$.

To prove (v), let $f \in \mathcal{A}$ and fix $\varepsilon>0$. By Definition 2.2 (iii) there exists a neighbourhood $V$ of the unit $e \in G$ such that the Haar measure $|V|$ of $V$ is finite and

$$
\left\|\mathcal{R}_{t} f-f\right\|_{\mathcal{A}}<\varepsilon \quad \text { for all } \quad t \in V \text {. }
$$


Let $g:=\frac{1}{|V|} \chi_{V} \in L_{\omega}^{1}(G)$. Note that

$$
g * f-f=\mathcal{R}_{g} f-f=\frac{1}{|V|} \int_{V}\left(\mathcal{R}_{t} f-f\right) d t .
$$

We have

$$
\|g * f-f\|_{\mathcal{A}} \leq \frac{1}{|V|} \int_{V}\left\|\mathcal{R}_{t} f-f\right\|_{\mathcal{A}} d t<\varepsilon .
$$

By Definition $2.2(\mathrm{v})$ we can find $h \in L_{\omega}^{1}(G) \cap \mathcal{A}$ such that $\|g-h\|_{\omega}<\varepsilon$. From (*) it follows that

$$
\|(g-h) * f\|_{\mathcal{A}}=\left\|\mathcal{R}_{g-h} f\right\|_{\mathcal{A}} \leq\|f\|_{\mathcal{A}} \cdot\|g-h\|_{\omega} .
$$

Thus for $h * f \in \mathcal{A}$ we have

$$
\|h * f-f\|_{\mathcal{A}} \leq\|g * f-f\|_{\mathcal{A}}+\|(g-h) * f\|_{\mathcal{A}}<\varepsilon\left(1+\|f\|_{\mathcal{A}}\right) .
$$

This implies (v).

Proposition 2.4 (iii) implies that an algebra $\mathcal{A}$ in the class $\mathcal{S}(G)$ satisfies Ditkin's condition at infinity. Next we will show that it satisfies Ditkin's condition at every point $\gamma \in \hat{G}=\Delta(\mathcal{A})$. For this we need some auxiliary results. First we recall that there exist left invariant means on $l^{\infty}(S)$ whenever $S$ is an abelian semigroup [7, p. 109, Theorem 4]. A left invariant mean on $l^{\infty}(S)$ is a bounded linear functional $\phi \in\left(l^{\infty}(S)\right)^{*}$ such that

(i) $\phi(f) \geq 0$ for all $f \geq 0$;

(ii) $\phi(\mathbf{1})=1$, where $\mathbf{1}$ is the constant one function;

(iii) $\phi(f(\cdot+s))=\phi(f)$ for all $f \in l^{\infty}(S)$ and $s \in S$.

Lemma 2.5. Let $\mathcal{A}$ be a Banach algebra in the class $\mathcal{S}(G)$. Let $\mathcal{B}, \mathcal{C}$ be two closed ideals of $\mathcal{A}$ with $\mathcal{C} \subset \mathcal{B}$. If $\gamma \in \hat{G}$ and $\psi \in(\mathcal{B} / \mathcal{C})^{*}$ satisfy

$$
\psi\left(\left[\mathcal{R}_{t} f\right]\right)=\gamma(t) \psi([f])
$$

for all $t \in G$ and $[f] \in \mathcal{B} / \mathcal{C}$, where $[f]$ denotes the equivalence class $f+\mathcal{C}$ in $\mathcal{B} / \mathcal{C}$, then $\psi([f])=0$ for all $f \in \mathcal{B} \cap k(\{\gamma\})$.

Proof. Let $K$ be the constant and $S$ be the subsemigroup corresponding to the semi-bounded weight $\omega$ given by Definition 2.2. By the Hahn-Banach theorem we can extend $\psi$ to an element $\psi \in(\mathcal{A} / \mathcal{C})^{*}$. Let $\phi$ be an invariant mean on $l^{\infty}(S)$. Since

$$
\sup \left\{\left\|\mathcal{R}_{s}\right\|_{\mathcal{A}}: s \in S\right\} \leq K<\infty
$$

by Definition 2.2 (iv), for each $f \in \mathcal{A}$ the function

$$
F(f ; s):=\gamma(s)^{-1} \psi\left(\left[\mathcal{R}_{s} f\right]\right), \quad s \in S,
$$

is in $l^{\infty}(S)$, and thus

$$
\Psi(f):=\phi(F(f ; \cdot)), \quad f \in \mathcal{A},
$$

defines a linear functional on $\mathcal{A}$ with $\|\Psi\| \leq K\|\psi\|$. For $s, t \in S$ and $f \in \mathcal{A}$ we have

$$
F\left(\mathcal{R}_{s} f ; t\right)=\gamma(s) \cdot\left(\gamma(s+t)^{-1} \psi\left(\left[\mathcal{R}_{s+t} f\right]\right)\right)=\gamma(s) F(f ; s+t) .
$$

It follows that

$$
\Psi\left(\mathcal{R}_{s} f\right)=\gamma(s) \phi(F(f ; \cdot+s))=\gamma(s) \Psi(f)
$$


where in the last equality we use the left invariance of $\phi$. In other words, $\mathcal{R}_{s}^{*} \Psi=$ $\gamma(s) \Psi$ for all $s \in S$. Applying $\mathcal{R}_{-s}^{*}$ to both sides, we also have $\mathcal{R}_{-s}^{*} \Psi=\gamma(-s) \Psi$, and therefore $\mathcal{R}_{t}^{*} \Psi=\gamma(t) \Psi$ for all $t \in G$, since $S-S=G$. Thus

$$
\Psi\left(\mathcal{R}_{t} f\right)=\gamma(t) \Psi(f) \quad \text { for all } t \in G, f \in \mathcal{A} .
$$

For $g \in L_{\omega}^{1}(G) \cap \mathcal{A}$ and $f \in \mathcal{A}$ this implies

$$
\begin{aligned}
\Psi(g * f) & =\Psi\left(\mathcal{R}_{g} f\right)=\int_{G} \Psi\left(\mathcal{R}_{t} f\right) g(t) d t \\
& =\int_{G} \gamma(t) \Psi(f) g(t) d t=\hat{g}(\gamma) \Psi(f) .
\end{aligned}
$$

By Definition $2.2(\mathrm{v}), L_{\omega}^{1}(G) \cap \mathcal{A}$ is norm dense in $\mathcal{A}$. Therefore,

$$
\Psi(g * f)=\hat{g}(\gamma) \Psi(f) \quad \text { for all } \quad g, f \in \mathcal{A} .
$$

Let $g \in k(\{\gamma\}) \cap \mathcal{B}$. Then, $\Psi(g * f)=0$ for all $f \in \mathcal{A}$. By Proposition $2.4(\mathrm{v}), g$ is in the norm closure of $g * \mathcal{A}$. Hence $\Psi(g)=0$. Our assumptions on $\gamma$ and $\psi$ imply

$$
F(g ; s)=\gamma(s)^{-1} \psi\left(\left[\mathcal{R}_{s} g\right]\right)=\psi([g])
$$

for all $s \in S$. Thus

$$
0=\Psi(g)=\phi(F(g ; \cdot))=\psi([g]),
$$

which completes the proof.

Next we recall an extension of Gelfand's theorem due to Atzmon [2]: $\quad$ If $T \in$ $\mathcal{L}(X)$ is invertible and satisfies

$$
\sup _{n \geq 1}\left\|T^{n}\right\|<\infty, \quad \lim _{n \rightarrow+\infty} \frac{\log ^{+}\left\|T^{-n}\right\|}{\sqrt{n}}=0,
$$

then $\sigma(T)=\left\{z_{0}\right\}$ implies $T=z_{0} I_{X}$.

Lemma 2.6. Let $\omega$ be a semi-bounded weight on the $L C A$ group $G$ and let $T: G \rightarrow$ $\mathcal{L}(X)$ be a strongly continuous group representation such that $\left\|T_{t}\right\| \leq \omega(t)$ for all $t \in G$. If $\gamma \in \operatorname{Sp}(T)$ is an isolated point, then there exists $0 \neq x^{*} \in X^{*}$ such that

$$
T_{t}^{*} x^{*}=\gamma(t) x^{*} \quad \text { for all } t \in G \text {. }
$$

Proof. Let $T: M_{\omega}(G) \rightarrow \mathcal{L}(X)$ be the extension of the given group representation $T$. Let $f_{0} \in L_{\omega}^{1}(G)$ be such that $\hat{f}_{0}(\gamma)=1$ and $\operatorname{supp} \hat{f}_{0}$ is disjoint from $\operatorname{Sp}(T) \backslash\{\gamma\}$. Then $f_{0}-f_{0} * f_{0} \in j_{\omega}(\operatorname{Sp}(T))$, and thus, by Theorem 1.1 (i),

$$
0=T_{f_{0}-f_{0} * f_{0}}=T_{f_{0}}-\left(T_{f_{0}}\right)^{2} .
$$

Hence, the operator $P:=T_{f_{0}}$ is a projection, and for the spectrum of the subspace representation $T \mid P X$ we have

$$
\operatorname{Sp}(T \mid P X)=\{\gamma\} .
$$

From the Spectral Mapping Theorem 1.1 (ii) applied to $T \mid P X$ we conclude that

$$
\sigma\left(T_{t} \mid P X\right)=\{\gamma(t)\} \quad \text { for all } t \in G .
$$

Let $K$ be the constant and $S$ be the subsemigroup corresponding to $\omega$. Then, for $s \in S$ we have

$$
\left\|T_{n s} \mid P X\right\| \leq K \quad \text { and } \quad \log ^{+}\left\|T_{-n s} \mid P X\right\|=o(\sqrt{n})
$$


as $n \rightarrow+\infty$. Applying Atzmon's theorem to the operators $T_{s} \mid P X$, we find that

$$
T_{s} P=\gamma(s) P \text {. }
$$

Since $S-S=G$, this identity holds for all $s \in G$. It follows that

$$
T_{t}^{*} P^{*}=\gamma(t) P^{*} \quad \text { for all } \quad t \in G \text {. }
$$

Choose $y^{*} \in X^{*}$ such that $x^{*}:=P^{*} y^{*} \neq 0$. Then

$$
T_{t}^{*} x^{*}=\gamma(t) x^{*} \quad \text { for all } \quad t \in G \text {. }
$$

A slightly more general result is proved in [11, Theorem 3.1.8].

Our main result now reads as follows.

Theorem 2.7. Let $\mathcal{A}$ be a Banach algebra in the class $\mathcal{S}(G)$. Let $\Lambda \subset \hat{G}$ be a closed subset whose boundary $\partial \Lambda$ is scattered. Assume $f \in k(\Lambda) \cap \mathcal{A}$. Then,

$$
f \in \overline{f *(j(\Lambda) \cap \mathcal{A})} .
$$

As a consequence, $\Lambda$ is a spectral set for the Banach algebra $\mathcal{A}$ in the sense that

$$
k(\Lambda) \cap \mathcal{A}=\overline{j(\Lambda) \cap \mathcal{A}}
$$

and $\mathcal{A}$ is a Ditkin algebra.

Proof. Consider

$$
\mathcal{B}:=\overline{f * \mathcal{A}} \quad \text { and } \quad \mathcal{C}:=\overline{f *(j(\Lambda) \cap \mathcal{A})}
$$

Then $\mathcal{B}$ and $\mathcal{C}$ are translation-invariant ideals of $\mathcal{A}$ with $\mathcal{C} \subseteq \mathcal{B}$. By Proposition 2.4

(v) we have $f \in \mathcal{B}$. We will show that $f \in \mathcal{C}$ by proving that $\mathcal{B}=\mathcal{C}$.

Consider the quotient space $X:=\mathcal{B} / \mathcal{C}$. Let $\omega$ be a semi-bounded weight as in Definition 2.2. The translation representation $\mathcal{R}$ of $G$ on $\mathcal{A}$ derives a strongly continuous group representation $Q$ of $G$ on $X$ by

$$
Q_{t}[g]:=\left[\mathcal{R}_{t} g\right], \quad g \in \mathcal{B}, t \in G
$$

here we write $[g]$ for the class $g+\mathcal{C}$. Clearly,

$$
\left\|Q_{t}\right\|_{X} \leq\left\|\mathcal{R}_{t}\right\|_{\mathcal{A}} \leq \omega(t), \quad t \in G
$$

Furthermore,

$$
Q_{\mu}[g]=[\mu * g], \quad g \in \mathcal{B}, \mu \in M_{\omega}(G) .
$$

We will show that $X=\{0\}$ by checking that $Q$ has empty spectrum.

Assume for a contradiction that $X \neq\{0\}$ and thus $\operatorname{Sp}(Q) \neq \emptyset$. To compute the spectrum of $Q$ we note that $Q_{g}=0$ for all $g \in j_{\omega}(\Lambda)$. It follows that

$$
\operatorname{Sp}(Q) \subseteq \mathrm{h}\left(j_{\omega}(\Lambda)\right)=\Lambda .
$$

We want to show that $\operatorname{Sp}(Q)$ is contained in the boundary $\partial \Lambda$. To see this, let $\gamma$ be an interior point in $\Lambda$. Then, by the regularity of $L_{\omega}^{1}(G)$, we can find a function $g_{\gamma} \in L_{\omega}^{1}(G)$ such that $\hat{g}_{\gamma}(\gamma)=1$ and $\operatorname{supp} \hat{g}_{\gamma} \subseteq \Lambda \backslash \partial \Lambda$. Since $\hat{f}$ vanishes on $\Lambda$, we have $\hat{f} \cdot \hat{g}_{\gamma}=0$, and thus $f * g_{\gamma}=0$ by the uniqueness of the Fourier transform. Therefore, $\mathcal{R}_{g_{\gamma}} f=f * g_{\gamma}=0$, and thus $Q_{g_{\gamma}}[f]=\left[\mathcal{R}_{g_{\gamma}} f\right]=0$. This implies $\gamma \notin \operatorname{Sp}(Q)$, and hence

$$
\operatorname{Sp}(Q) \subseteq \partial \Lambda
$$


Therefore $\operatorname{Sp}(Q)$ is a non-empty scattered set, since $\partial \Lambda$ is scattered by assumption. Let $\gamma_{0}$ be an isolated point in $\operatorname{Sp}(Q)$. Applying Lemma 2.6 to $Q$, we find $0 \neq \psi \in X^{*}$ such that $Q_{t}^{*} \psi=\gamma_{0}(t) \psi$ for all $t \in G$, i.e.,

$$
\psi\left(Q_{t}[g]\right)=\gamma_{0}(t) \psi([g])
$$

for all $t \in G$ and $[g] \in X$. Lemma 2.5 yields

$$
\psi([g])=0 \quad \text { for all } \quad g \in k\left(\left\{\gamma_{0}\right\}\right) \cap \mathcal{B} .
$$

Note that $f * h \in k\left(\left\{\gamma_{0}\right\}\right) \cap(f * \mathcal{A}) \subseteq k\left(\left\{\gamma_{0}\right\}\right) \cap \mathcal{B}$ for all $h \in \mathcal{A}$. Thus $\psi([f * h])=0$ for all $h \in \mathcal{A}$, and hence $\psi=0$, a contradiction.

This proves the first assertion. It follows that $\mathcal{A}$ satisfies Ditkin's condition at every point $\gamma \in \hat{G}$. As we have seen before, $\mathcal{A}$ also satisfies Ditkin's condition at infinity. Hence, $\mathcal{A}$ is a Ditkin algebra.

Corollary 2.8. Let $\omega$ be a semi-bounded weight on the LCA group G. Then the Beurling algebra $L_{\omega}^{1}(G)$ is a Ditkin algebra.

It is shown in [17, pp. 92-93] that all Segal algebras are Ditkin algebras. Since each Segal algebra on $G$ belongs to the class $\mathcal{S}(G)$ (see Example 2.3), our Theorem 2.7 gives a functional analytic proof of this result.

Our final result is an application of [5, Theorem 2.3] to algebras in $\mathcal{S}(G)$.

Proposition 2.9. Let $\mathcal{A}$ be a Banach algebra in the class $\mathcal{S}(G)$ and $I$ be a closed ideal in $\mathcal{A}$. Then each epimorphism from a Banach algebra onto $\mathcal{A} / I$ is automatically continuous. As a result, the algebra $\mathcal{A} / I$ has a unique complete norm.

Note that non-semisimple quotients arise when $\mathcal{A}=L^{1}(G)$ where $G$ is a nondiscrete LCA group and $I=\overline{j(\Lambda)}$ for some non-spectral set $\Lambda \subset \hat{G}$. The existence of such ideals is the well-known theorem of Malliavin [15]. In this situation Johnson's uniqueness theorem for semisimple Banach algebras cannot be applied.

\section{REFERENCES}

[1] W. Arveson: On groups of automorphisms of operator algebras. J. Funct. Anal. 15 (1974), 217-243. MR 50:1016

[2] A. Atzmon: Operators which are annihilated by analytic functions. Acta Math. 144 (1980), 27-63. MR 81c: 47007

[3] J. J. Benedetto: "Spectral Synthesis," B.G. Teubner, Stuttgart (1975). MR 58:29850a

[4] A. Beurling: Sur les intégrales de Fourier absolument convergentes, IX ${ }^{e}$ Congrès Math. Scand., Helsinki, pp. 345-366 (1966).

[5] W. G. Bade and H. G. Dales: Uniqueness of complete norms for quotients of Banach function algebras. Studia Math. 106 (1993), 289-302. MR 94f: 46062

[6] C. D'Antoni, C. Longo And L. Zsidó: A spectral mapping theorem for locally compact groups of operators. Pacific J. Math. 103 (1982), 17-24. MR 84e:47058

[7] M. M. DAY: "Normed Linear Spaces," 3rd ed., Springer-Verlag, Berlin- Heidelberg-New York (1973). MR 49:9588

[8] V. Ditkin: On the structure of ideals in certain normed rings. Uchen. Zap. Mosk. Gos. Univ. Matem. 30 (1939), 81-130. (Russian; English summary) MR 1:336b

[9] Y. Domar: Harmonic analysis based on certain commutative Banach algebras. Acta Math. 96 (1956), 1-66. MR 17:1228a

[10] E. Hewitt and R. Ross: "Abstract Harmonic Analysis I," Springer-Verlag, BerlinHeidelberg-New York (1963). MR 28:158

[11] S.-Z. HuAng: "Spectral Theory for Non-Quasianalytic Representations of Locally Compact Abelian Groups," Thesis, Universität Tübingen (1996). A summary has appeard in "Dissertation Summaries in Mathematics" 1 (1996), 171-178. 
[12] Y. Katznelson: "An Introduction to Harmonic Analysis," 2nd ed., Dover Publications, New York (1976). MR 54:10976

[13] R. Larsen, T. S. LiU And J. K. Wang: On functions with Fourier transforms in $L_{p}$. Michigan Math. J. 11 (1964), 369-378. MR 30:412

[14] L. H. Loomis: "An Introduction to Abstract Harmonic Analysis," van Nostrand, New York (1953). MR 14:883c

[15] P. Malliavin: Impossibilité de la synthèse spectrale sur les groupes abéliens. Inst. Hautes Études Sci. Publ. Math. 2 (1959), 61-68. MR 21:5854c

[16] R. Reiter: "Classical Harmonic Analysis and Locally Compact Groups," Oxford Univ. Press, London (1968). MR 46:5933

[17] R. Reiter: " $L^{1}-$ Algebras and Segal Algebras," Springer-Verlag, Berlin-Heidelberg-New York (1971). MR 55:13158

[18] I. E. Segal: The group algebra of a locally compact group. Trans. Amer. Math. Soc. 61 (1947), 69-105. MR 8:438c

[19] M. WolfF: Spectral theory of group representations and their nonstandard hull. Israel J. Math. 48 (1984), 205-224. MR 86e:46046

[20] M. Zarrabi: Ensembles de synthèse pour certaines algèbres de Beurling. Rev. Roumaine Math. Pures Appl. 35 (1990), 385-396. MR 92a:43005

Mathematisches Institut, Universität Tübingen, Auf der Morgenstelle 10, D-72076 Tübingen, F. R. Germany

Current address, Sen-Zhong Huang: Mathematisches Institut, Friedrich-Schiller-Universität Jena, Ernst-Abbe-Platz 1-4, D-07743 Jena, Germany

E-mail address: huang@mipool.uni-jena.de

Current address, Jan van Neerven: Department of Mathematics, Tu Delft, P. O. Box 356, 2600

AJ Delft, the Netherlands

E-mail address: J.vanNeerven@twi.tudelft.nl

E-mail address: frra@michelangelo.mathematik.uni-tuebingen.de 